\title{
Evolutions temporelles de la concentration en gaz à effet de serre et du climat d'après la carotte de Vostok (Antarctique)
}

\author{
Time evolutions of the greenhouse effect gases concentration \\ and the climate from the Vostok core
}

par Laurence Pépin, Jean-Marc Barnola, Jean-Robert Petit, Dominique Raynaud

Laboratoire de Glaciologie et de Géophysique de l'Environnement du CNRS, Associé à l'Université Joseph Fourier, Grenoble

Polar ice and air bubbles trapped in it, attest climate history and the atmospheric composition of the Earth. The Vostok drill at the Russian station of Vostok on the Antarctic Plateau provides a more 400000 -year long record of these conditions. Through this time, climate in Antarctica oscillated between 4 glacials, for which surface temperatures were about $10^{\circ} \mathrm{C}$ below modern ones, and 5 interglacial periods as the modern one. It is shown that greenhouse gases $\left(\mathrm{CO}_{2}\right.$ and $\left.\mathrm{CH}_{4}\right)$ concentrations are higher during warm periods than cold ones. Scrutinising glacial to interglacial transitions shows that Southern Hemisphere processes lead northern ones. Furthermore, sea level rise appears to lag variations of other variables. Finally, these records give evidence that $\mathrm{CO}_{2}$ and $\mathrm{CH}_{4}$ levels measured at present time, have never been reached during the late 420000 years.

\section{I - L'ARCHIVAGE GLACIAIRE}

La neige qui se dépose à la surface des régions polaires a une composition isotopique qui caractérise sa température de formation. En tombant, les flocons lessivent l'atmosphère et incorporent alors des impuretés (aérosols) fournissant ainsi des informations sur l'état de l'atmosphère. Ensuite, la neige se transforme lentement en glace, passant d'un milieu à porosité ouverte, c'est-à-dire en contact avec l'atmosphère, à un milieu isolé dont $10 \%$ environ du volume est composé de bulles d'air [1]. La durée du processus de densification du névé polaire implique que l'air soit plus jeune que la glace qui l'entoure. La connaissance de cette différence d'âge est importante pour comparer les enregistrements de la composition gazeuse de l'atmosphère aux paramètres climatiques issus de la glace elle-même. De plus, il faut tenir compte des processus physiques (gravitation, diffusion) qui peuvent modifier légèrement la composition de l'air du névé et donc des bulles, pour connaître les concentrations passées des divers gaz. Cela suppose une bonne connaissance des paramètres profondeur et température, correspondant au niveau de fermeture des pores. Dans le cas de l'étude des gaz atmosphériques, il est également nécessaire de s'affranchir des processus générés par la présence de couches de fusion en surface, de fractures ou encore par des contenus relativement importants en impuretés (cas de la glace du Groënland) qui, par réactions chimiques, peuvent modifier la composition initiale en $\mathrm{CO}_{2}$.

\section{VOSTOK : UN SITE IDÉAL POUR LES ÉTUDES PALÉO-CLIMATIQUES À LONG TERME}

Le site de Vostok en Antarctique de l'Est (78 $28^{\prime}$ Sud, $106^{\circ} 48^{\prime}$ Est ; $3488 \mathrm{~m}$ d'altitude) est particulièrement indiqué pour les études paléo-climatiques sur de longues périodes. Il y règne en effet une température si basse (jusqu'à $-89^{\circ} \mathrm{C}$ en hiver et environ $-55^{\circ} \mathrm{C}$ en moyenne annuelle) que toute fusion même partielle des couches de surface est exclue, garantissant ainsi l'intégrité des enregistrements gazeux et en particulier celui du $\mathrm{CO}_{2}$. De plus le contenu en impuretés de la neige déposée dans cette région centrale de l'Antarctique de l'Est est particulièrement faible. Enfin, les précipitations sont rares sur cette partie de l'Antarctique. A Vostok l'accumulation moyenne annuelle est d'environ $2,3 \mathrm{~g} / \mathrm{cm}^{2} / \mathrm{an}$, ce qui correspond à moins de $10 \mathrm{~cm}$ de précipitation neigeuse par an. Cette faible accumulation conjuguée à l'épaisseur de la calotte de glace qui est de plus de $3700 \mathrm{~m}$, font du site de Vostok le lieu idéal pour reconstruire de manière la plus directe possible, l'histoire du climat et de l'atmosphère de notre planète sur une très longue période.

2.1 Historique des opérations de forage à Vostok

Installés à Vostok depuis l'année géophysique internationale (1957), les Soviétiques informent en 1982 la commu- 


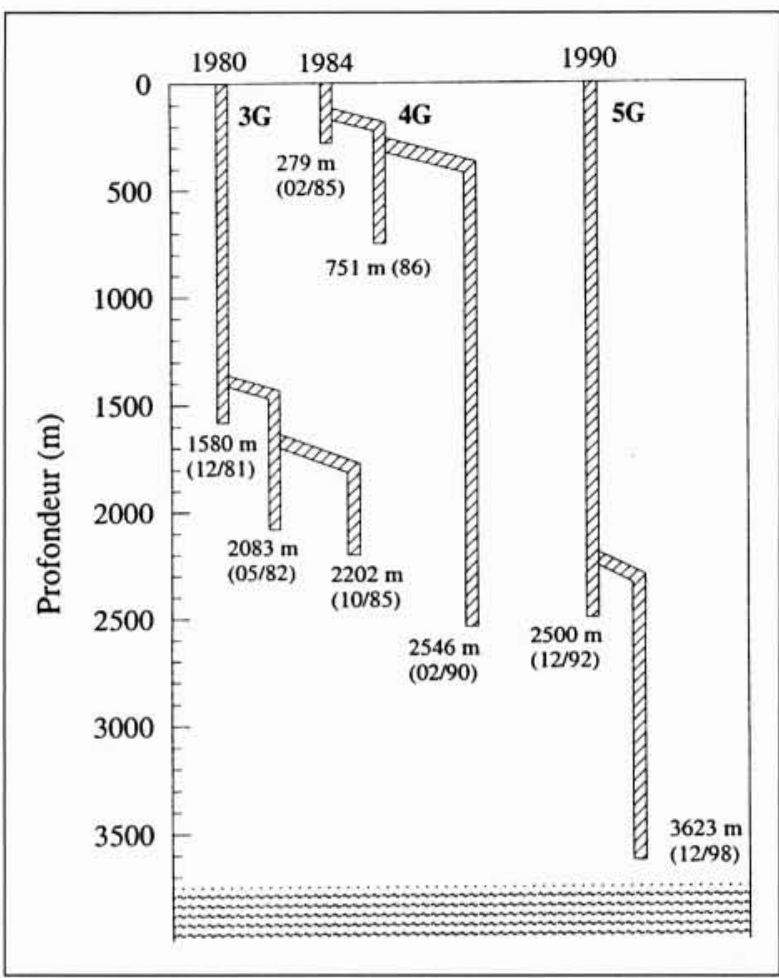

1. Historique des opérations de forages profonds à la station de Vostok depuis 1980 (adapté de Jouzel et al., Climate Dynamics, vol. 12, 1996).

nauté scientifique internationale que le forage $3 \mathrm{G}$ a atteint la profondeur de $2082 \mathrm{~m}$ (voir l'historique des forages sur la figure 1). Dès lors, une collaboration scientifique s'engage entre la France et l'Union Soviétique qui aboutit en 1985 à la publication de premiers résultats portant sur un cycle climatique complet [2]. En 1989 les Etats-Unis se joignent à la Russie et la France pour poursuivre l'étude des carottes du forage profond à Vostok et en 1990 le puits n 5 est amorcé. Vostok est alors un projet tripartite tant sur le plan technique et logistique, que scientifique. La France contribue à ces opérations par le biais de l'Institut Français de Recherche en Terres Polaires (IFRTP) pour la partie logistique, alors que le volet scientifique est confié principalement à deux laboratoires : le Laboratoire de Glaciologie et de Géophysique de l'Environnement (LGGE, Grenoble) et le Laboratoire des
Sciences du Climat et de l'Environnement (LSCE, Saclay). En 1993, alors que le forage atteint la profondeur de $2755 \mathrm{~m}$, la station ferme pour l'hiver. Les opérations se poursuivent alors uniquement pendant les périodes estivales et en fin de saison 1998 le forage est stoppé à la profondeur record de $3623 \mathrm{~m}$. Cette profondeur ne correspond pas à celle du socle rocheux, puisque environ $120 \mathrm{~m}$ plus bas s'étend un lac sous-glaciaire. Mais certaines perturbations des enregistrements, dues à l'écoulement de la glace et à la présence du lac, sont suspectées au-dessous de $3310 \mathrm{~m}$ de profondeur $[3,4]$. Par conséquent nous présenterons ici les enregistrements climatiques issus des $3310 \mathrm{~m}$ supérieurs de la carotte, ce qui représente l'enregistrement continu de la composition de l'atmosphère le plus long existant à ce jour puisqu'à cette profondeur la glace est vieille de plus de 420000 ans [3].

\subsection{Quelques paramètres mesurés à Vostok et leur signification climatique}

Il existe deux catégories de paramètres que l'on peut extraire des carottes de glace. D'une part les informations de la matrice de glace elle-même, d'autre part les concentrations en différents gaz et de leurs isotopes que l'on mesure à partir de l'analyse de l'air piégé dans les bulles d'air au sein de la glace.

Dans le médium "glace" on mesure en particulier la composition isotopique de la glace (teneur en deutérium dans le cas de Vostok). Cette variable est notée $\delta D$, elle est associée à la température de formation des précipitations c'est-à-dire à une centaine de mètres d'altitude au-dessus de la surface. Finalement, on relie la teneur en deutérium de la glace à la température de surface.

On mesure également dans la glace la concentration en particules continentales insolubles. La quantité de poussières parvenant à Vostok dépend des conditions environnementales régnant en Amérique du Sud (Patagonie), région d'origine de ces poussières, ainsi que du transport de celle-ci jusqu'en Antarctique. L'expression "conditions environnementales" recouvre les conditions d'aridité, de rugosité des sols, de couverture végétale, de vitesse et fréquence des vents de surface... qui caractérisent cette région. On suggère par ailleurs que l'étendue de la glace de mer en Atlantique Sud semble jouer un rôle important dans les variations de ce paramètre.

A partir de l'analyse de l'air contenu dans la glace on déduit en particulier les variations de la teneur en $\mathrm{CO}_{2}$ et en

\begin{tabular}{|l|l|l|}
\hline Média & Paramètre mesuré & Signification climatique ou environnementale \\
\hline \multirow{2}{*}{ Glace } & $\delta \mathrm{D}(\%)$ & Température en Antarctique \\
\cline { 2 - 3 } & Poussières & Conditions environnementales en Amérique du Sud (Patagonie) \\
\hline \multirow{2}{*}{$\mathrm{Gaz}$} & $\begin{array}{l}\mathrm{CO}_{2}(\mathrm{ppmv}: \text { parties par million } \\
\text { en volume) }\end{array}$ & $\begin{array}{l}\text { Effet radiatif } \\
\text { Cycle du carbone }\end{array}$ \\
\cline { 2 - 3 } & $\begin{array}{l}\mathrm{CH}_{4}(\mathrm{ppbv}: \text { parties par million } \\
\text { en volume) }\end{array}$ & $\begin{array}{l}\text { Effet radiatif } \\
\text { Cycle du carbone (continents) } \\
\text { Réchauffement de l'hémisphère Nord } \\
\text { Déglaciation rapide de l'hémisphère Nord }\end{array}$ \\
\cline { 2 - 3 } & $\delta^{18} \mathrm{O}_{\text {atm }}(\%)$ & $\begin{array}{l}\text { Volume global des glaces continentales } \\
\text { Cycle hydrologique aux basses latitudes }\end{array}$ \\
\hline
\end{tabular}

Tableau I. Paramètres mesurés dans la carotte de Vostok. 


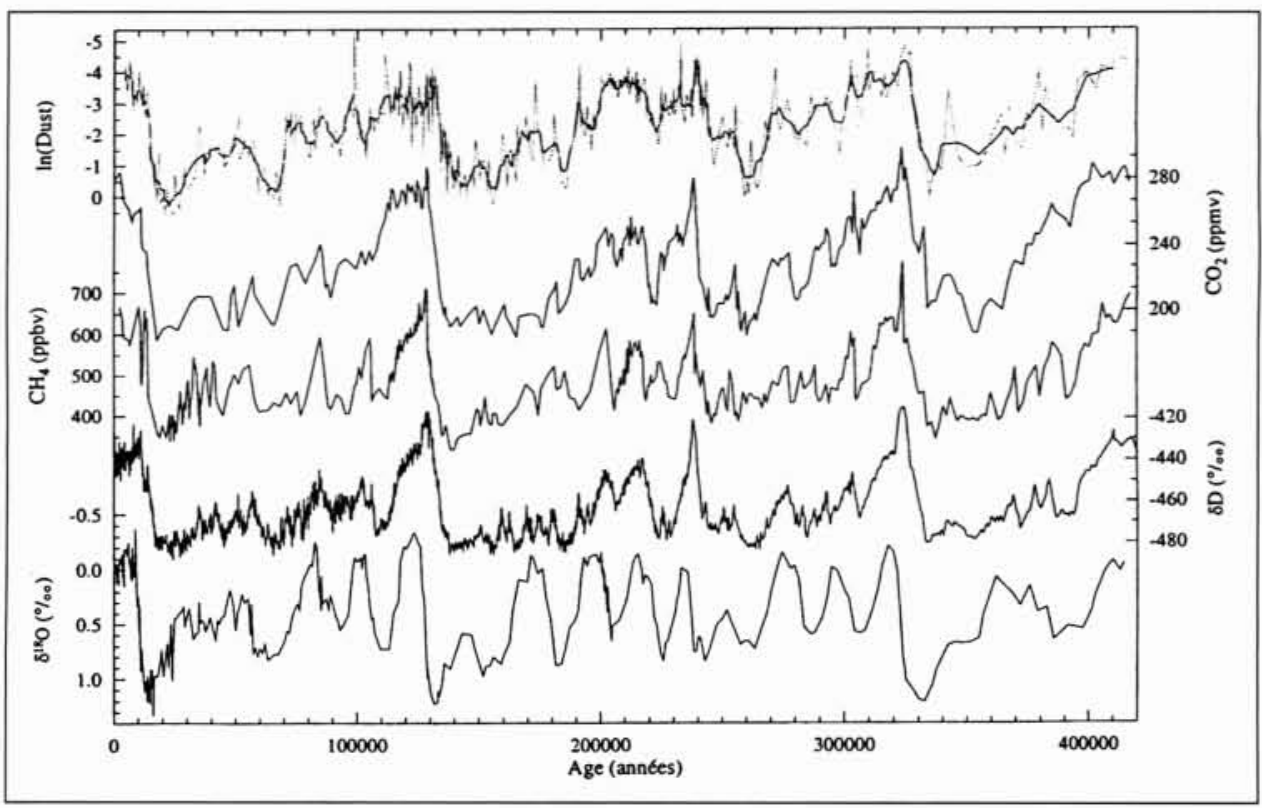

2. Evolutions temporelles des poussières, du $\mathrm{CO}_{2}$, du $\mathrm{CH}_{4}$, du $\delta \mathrm{D}$ (température) et du $\delta^{18} \mathrm{O}_{\text {atm }}$ au cours des 420000 dernières années (adaptée de [3]).

$\mathrm{CH}_{4}$ de l'atmosphère. Ces gaz sont après la vapeur d'eau, et par ordre décroissant d'importance, les 2 principaux gaz à effet de serre. Cela signifie que ces molécules possèdent la propriété de capter l'énergie émise par la Terre dans les grandes longueurs d'ondes et d'empêcher ainsi sa dissipation vers l'espace. Ce mécanisme contribue au réchauffement de la planète ; à ce titre il est important de comprendre les processus qui font évoluer de manière naturelle la concentration de ces gaz dans l'atmosphère. De plus, $\mathrm{CO}_{2}$ et $\mathrm{CH}_{4}$ sont répartis de manière particulièrement homogène dans l'atmosphère. L'enregistrement du $\mathrm{CO}_{2}$ dans les glaces de l'hémisphère Nord n'est pas fiable en raison de réactions chimiques rendues possibles par la forte concentration d'impuretés de cette glace. En revanche, l'enregistrement du $\mathrm{CH}_{4}$ n'est pas faussé par ce biais ; on se sert alors des caractéristiques de celui-ci (accélération de l'augmentation de la concentration du $\mathrm{CH}_{4}$ lors des transitions glaciaire-interglaciaires) comme marqueurs stratigraphiques des changements climatiques de l'hémisphère Nord (réchauffement, influence sur le volume des glaces continentales) et pour les repérer dans les enregistrements de l'hémisphère Sud.

Les variations de la teneur en isotope 18 de l'oxygène des bulles d'air $\left(\delta^{18} \mathrm{O}_{\text {atm }}\right)$ dépendent de 2 facteurs principaux : d'une part du cycle hydrologique aux basses latitudes et d'autre part des variations du volume des glaces continentales (donc du niveau marin). Par rapport au volume des glaces, il faut toutefois tenir compte d'un retard du $\delta^{18} \mathrm{O}_{\text {atm }}$ d'environ 2000 ans lié au transfert du signal océanique vers l'atmosphère via la photosynthèse.

Le résumé des paramètres mesurés dans la carotte de Vostok et les significations que nous leur attribuons sont donnés dans le tableau 1.

\subsection{Quatre cycles climatiques révélés par la carotte de Vostok}

Sur la figure 2 sont présentées les évolutions temporelles de la température, des poussières (échelle logarithmique), du $\mathrm{CO}_{2}$, du $\mathrm{CH}_{4}$ et du $\delta^{18} \mathrm{O}_{\text {atm }}$, au cours des 420000 dernières années. Les données et la chronologie utilisées sont celles publiées par Petit et al. [3].

Les paramètres du climat et de l'environnement montrent 4 cycles majeurs appelés cycles glaciaire-interglaciaires avec des profils caractéristiques en "dents de scie". Les enregistrements sont marqués par des périodicités de 100000 et 40000 ans, sauf celui du $\delta^{18} \mathrm{O}_{\text {atm }}$ qui est dominé par une composante de 20000 ans. A la manière de celles observées dans les sédiments marins, ces périodes témoignent de la modulation du climat par les variations séculaires de l'insolation associées aux changements de l'orbite de la Terre autour du Soleil (théorie de Milankovitch).

Pendant ces 4 grands cycles climatiques, les caractéristiques de l'atmosphère ont varié entre des limites relativement stables : l'amplitude des variations glaciaireinterglaciaires de la température en Antarctique est de l'ordre de $12^{\circ} \mathrm{C}$ au niveau du sol ; entre les périodes glaciaires et interglaciaires les concentrations de l'atmosphère en gaz à effet de serre varient de $180 \mathrm{ppmv}$ à $280 \mathrm{ppmv}$ pour le $\mathrm{CO}_{2}$ et de 350 ppbv à 700 ppbv pour le $\mathrm{CH}_{4}$. Par rapport au climat actuel, les interglaciaires précédents (vers $-130000,-240000$ et -320000 ans) diffèrent en durée et en morphologie. La période chaude (Holocène) que nous connaissons depuis 10000 ans est celle qui présente la durée de stabilité la plus longue. En revanche les interglaciaires vers -130000 et -320000 ans auraient été plus chauds $\left(2^{\circ} \mathrm{C}\right.$ environ) pendant quelques milliers d'années.

Les concentrations en gaz à effet de serre apparaissent fortement corrélées à la température en Antarctique sur l'ensemble de la période étudiée, ce qui confirme les résultats précédemment publiés sur les derniers 150000 ans. Notons tout de même dans le cas du $\mathrm{CO}_{2}$ que celui-ci commence à diminuer en retard de quelques milliers d'années sur la température Antarctique lors des transitions vers les périodes glaciaires. Une hypothèse pour expliquer ce décalage est d'envisager la persistance de la biosphère continentale malgré le refroidissement au début des glaciations. On remarque finalement que les concentrations actuelles en $\mathrm{CO}_{2}$ et en $\mathrm{CH}_{4}$ (365 ppmv et $1700 \mathrm{ppbv}$ respectivement) n'ont jamais été atteintes au cours des 420000 dernières années, ce qui ne peut que nous interpeller quant à la contribution 
possible de ces gaz au futur réchauffement de notre planète.

On observe également une très forte ressemblance entre les profils du $\mathrm{CO}_{2}$ et de la concentration en poussières (échelle logarithmique). Cette corrélation est d'autant plus intéressante qu'elle est aussi valable lors des entrées en glaciation. La glace de mer, en réduisant par exemple les échanges entre l'atmosphère et l'océan, peut influencer la teneur en $\mathrm{CO}_{2}$ de l'atmosphère. Nous pensons que la glace de mer en Atlantique Sud affecte le climat de l'Amérique du Sud, ce qui peut ensuite jouer un rôle important dans l'érosion continentale, la production de poussières et leur transport jusqu'en Antarctique.

\subsection{Séquence des événements enregistrés à Vostok lors des transitions glaciaire-interglaciaires}

Pour recueillir plus d'informations sur les mécanismes susceptibles d'engendrer les oscillations glaciaire-interglaciaires du climat, il est intéressant d'examiner plus en détail la séquence des événements lors des transitions glaciaireinterglaciaires (autour de $-15000,-130000,-240000$ et 330000 ans).

De ce travail il ressort que l'enchainement des modifications glaciaire-interglaciaires de chacun des paramètres étudié est le même pour les 4 dernières transitions climatiques (aux incertitudes analytiques et de datation près). De manière générale lors des déglaciations, la séquence est la suivante :

- Diminution des poussières,

- Début de l'augmentation du $\mathrm{CH}_{4}(-600-+1100$ ans),

- Augmentation du $\mathrm{CO}_{2}$ (1100 - 1700 ans),

- Augmentation de la température (800 - 2600 ans),

- Diminution de $\delta^{18} \mathrm{O}_{\text {atm }}$ (3600 - 7300 ans),

- Accélération de l'augmentation du $\mathrm{CH}_{4}(4700$ - 10600 ans).

Les chiffres entre parenthèse sont les domaines de variation du retard de l'évolution de la variable considérée par rapport à la diminution des poussières qui est toujours le premier paramètre à réagir sauf pour la transition autour de 330000 ans.

L'analyse de cette séquence permet de tirer quelques conclusions quant aux mécanismes susceptibles d'expliquer les oscillations glaciaire-interglaciaires du climat et en particulier les variations de la teneur en $\mathrm{CO}_{2}$ de l'atmosphère.

Le quasi-synchronisme des variations du $\mathrm{CO}_{2}$ et du $\mathrm{CH}_{4}$ par rapport à la température antarctique est en accord avec l'hypothèse d'un rôle amplificateur des gaz à effet de serre vis-à-vis du réchauffement climatique. Notons à ce sujet que le retard apparent des modifications de la température antarctique sur la concentration de l'atmosphère en gaz à effet de serre (quelques centaines d'années) peut provenir de l'incertitude relative à l'évaluation de la différence d'âge entre la glace et le gaz. D'autre part, on s'aperçoit que les indicateurs des changements climatiques dans l'hémisphère Sud (température et poussières) sont en avance sur ceux plus spécifiques de l'hémisphère Nord $\left(\delta^{18} \mathrm{O}_{\mathrm{atm}}\right.$ et $2^{\text {nde }}$ phase de l'augmentation de la concentration en $\mathrm{CH}_{4}$ ). Ce résultat met en relief l'importance des mécanismes prenant naissance dans l'hémisphère Sud, conformément à ce qui a déjà été suggéré dans d'autres études [5]. Concernant les variations glaciaires-interglaciaires de la teneur en $\mathrm{CO}_{2}$ de l'atmosphère, on sait que l'océan y joue un rôle prépondérant (le stock de carbone de l'océan étant actuellement 60 fois plus important que celui de l'atmosphère). En revanche ce type d'étude semble montrer que les changements du volume de l'océan n'est pas l'élément initiateur des variations du $\mathrm{CO}_{2}$ dans l'atmosphère. En effet, on constate que les indicateurs du niveau marin (déduit de l'accélération de l'augmentation du $\mathrm{CH}_{4}$ qui est en phase avec une fonte marquée des glaces de l'hémisphère Nord, ou encore de la diminution du $\delta^{18} \mathrm{O}_{\mathrm{atm}}$ ) réagissent toujours en retard sur les variations du $\mathrm{CO}_{2}$ lors des 4 dernières transitions glaciaire-interglaciaires

\section{III - IMPLICATIONS ET TRAVAUX À VENIR}

Parmi les résultats que nous avons énoncés, il nous semble important d'insister sur quelques points. La séquence des événements suggère que l'augmentation de la concentration de ces gaz dans l'atmosphère soit en phase avec l'augmentation de la température antarctique dans la limite des incertitudes de l'enregistrement. Il semble de plus que ces augmentations aient lieu bien avant le grand réchauffement des hautes latitudes Nord et probablement avant la hausse du niveau marin. Finalement, il paraît maintenant certain que les teneurs actuellement mesurées de deux des principaux gaz à effet de serre, le $\mathrm{CO}_{2}$ et le $\mathrm{CH}_{4}$, n'ont jamais été atteintes au cours des 420000 dernières années.

Dans l'avenir le forage profond de Dôme C ( $74^{\circ} 39^{\prime}$ Sud, $124^{\circ} 1^{\prime}$ 'Est, altitude $3240 \mathrm{~m}$ ), dans le cadre du projet européen EPICA devrait fournir un nouvel enregistrement glaciaire couvrant une période équivalente. Nous pourrons ainsi affiner les résultats obtenus à Vostok.

\section{Remerciements}

La dextérité des foreurs russes est à la base des résultats présentés ici. Merci donc à eux, aux Expéditions Antarctiques Russes, à l'IFRTP et la NSF (section Polar Research) pour leur support logistique. Ce travail est largement inspiré de l'article [3] paru dans la revue "Nature" en 1999, merci à nos co-auteurs.

\section{Références bibliographiques}

[1] Raynaud D., Barnola J.-M., Chappellaz J. (1995), Les glaces polaires : une mémoire de l'évolution des gaz à effet de serre, La Houille Blanche, 5, 123-125

[2] LoRIus et al. (1985), A 150,000-year climatic record from Antarctic ice, Nature, 316, 6029, 591-596.

[3] Perrt J.-R. et al. (1999), Climate and atmospheric history of the past 420000 years from the Vostok ice core, Antarctica, Nature, 399, 429. 436

[4] JouzEL et al. (1999), More than 200 meters of lake ice above subglacial Lake Vostok, Antarctica, Science, 286, 2138-2141.

[5] Broecker W.S., Henderson G.M. (1998). The sequence of events surrounding termination II and their implications for the cause of glacialinterglacial $\mathrm{CO}_{2}$ changes, Paleoceanography, 13, 4, 352-364. 